\title{
PLANIFICACIÓN DE LA PRODUCCIÓN EN PLANTAS TERMOSOLARES. MODELADO DEL CAMPO SOLAR ORIENTADO A DATOS
}

\author{
E.G. Cojocaru, J.M. Bravo, M.J. Vasallo y M.E. Gegúndez
}

Departamento de Ingeniería Electrónica, de Sistemas Informáticos y Automática, Universidad de Huelva emails: manuel.vasallo@diesia.uhu.es, caro@uhu.es, emilian-gelu.cojocaru@alu.uhu.es, gegundez@uhu.es

\section{Resumen}

Este trabajo se encuadra en el contexto de la planificación óptima de la producción en plantas termosolares de concentración con almacenamiento térmico que participan en un mercado diario de electricidad. Un elemento habitual las estrategias de planificación óptima es la utilización de un modelo detallado del campo solar de la planta. Este modelo estima la potencia térmica captada por el campo solar a partir del recurso solar incidente. Sin embargo, el desarrollo de un modelo detallado que se ajuste de forma adecuada el campo solar es una tarea laboriosa y compleja. Este trabajo propone una estrategia diferente que permite sustituir el modelo detallado por un modelo de inferencia basado en un histórico de datos. En síntesis, dado un nuevo valor del recurso solar, se consulta el histórico de recursos incidentes y potencias captadas en el pasado y mediante un algoritmo de predicción se estima la potencia captada correspondiente. La estrategia propuesta se aplica, en un contexto de simulación, a una planta basada en colectores cilindro-parabólicos de $50 \mathrm{MW}$ con almacenamiento térmico bajo la hipótesis de participación en el mercado diario español de energía. Los resultados muestran que la nueva estrategia proporciona resultados competitivos con respecto a la estrategia basada en modelo detallado. Por tanto, como conclusión se puede inferir que sustituir el modelo detallado del campo solar por un modelo basado en datos puede ser una alternativa viable en estrategias de planificación de la producción.

Palabras clave: Operación óptima de sistemas de potencia. Integración en el mercado de la energía. Modelado y simulación de sistemas de potencia.

\section{INTRODUCCIÓN}

La energía solar térmica de concentración (CSP, concentrating solar power) es una tecnología implantada en países como España y Estados Unidos donde ha sido promovida gracias a políticas activas por parte de sus gobiernos. Una planta basada en CSP se compone, habitualmente, de va- rios bloques, el campo solar ( $\mathrm{SF}$, solar field), el bloque de potencia (PB, power block) y un sistema de almacenamiento térmico (TES, thermal energy storage). El SF convierte la radiación solar en potencia térmica. En el tipo de planta termosolar más extendido [16] un conjunto de colectores cilindro-parabólicos (PTC, parabolic trough collector) captura la radiación solar por medio del calentamiento de un fluido (HTF, head transfer fluid). La potencia térmica se transfiere al bloque de potencia desde el HTF mediante un conjunto de intercambiadores de calor. El PB es el encargado de generar potencia eléctrica. Actualmente las plantas basadas en CSP se combinan con algún tipo de sistema TES o bien sistemas de respaldo basados en combustibles fósiles. Esta ampliación desacopla la capacidad de producción de la disponibilidad del recurso solar, confiriendo a dichas plantas un carácter denominado semi-gestionable. Esta característica permite trasladar la producción de las plantas desde periodos temporales de precios de compra bajos a periodos con precios de compra altos. Es posible, por tanto, plantear el problema de la planificación óptima de la producción (también llamada auto-programación óptima) y participar en mercados eléctricos con precios variables. Desde el punto de vista de los propietarios de las plantas, el problema de la planificación óptima, al participar en mercados desregularizados, se traduce en el objetivo de maximizar los beneficios obtenidos mediante la venta de energía. Para maximizar dichos objetivos los propietarios deben disponer de predicciones de los precios de la energía y de la capacidad de producción de la propia planta. Dicha capacidad depende claramente del recurso solar disponible. Además, los propietarios deben anticipar con antelación su planificación diaria de producción, corriendo el riesgo de sufrir penalizaciones económicas por desvíos respecto a dicha planificación.

La planificación de la producción en plantas CSP ha sido ampliamente tratada en la literatura. En [10] se propuso una estrategia basada en la herramienta SAM [2] y un modelo de optimización basado en programación lineal entera mixta (MILP, mixed integer linear programming). Otros ejemplos con planteamientos basados en MILP se pue- 
den consultar en [11] y [6]. En [13] se propuso combinar la capacidad de reprogramación que proporciona el control predictivo basado en modelo (MPC, model-based predictive control) y un modelo de programación entera mixta (MIP) como estrategia de planificación de la producción.

Uno de los elementos presente en las estrategias de planificación óptima de la producción en plantas CSP es la utilización de un modelo detallado del SF [4]. Dicho modelo toma la irradiancia directa normal (DNI, direct normal irradiance) presente en el campo y proporciona una estimación de la potencia térmica transferida al HTF.

Este trabajo propone sustituir la estimación de la potencia térmica basada en un modelo detallado del SF, por una estimación basada directamente en datos históricos. Asumiendo la existencia de un histórico de datos que almacena DNIs pasados y potencia térmica transferida al HTF, dado una nueva predicción de DNI, el método propuesto consulta el histórico y proporciona una estimación de la futura potencia térmica transferida. La principal ventaja del método es la sustitución del modelo detallado del SF por un algoritmo de estimación y una consulta a una base de datos. Nótese que el desarrollo y ajuste fino de un modelo detallado de un SF no es una tarea sencilla. Por otra parte, conforme la planta se va degradando con el paso del tiempo, dicho modelo debe ser actualizado. Con el fin de ilustrar la propuesta se ha desarrollado un caso de estudio basado en la simulación de una planta de $50 \mathrm{MW}$, con PTC y TES bajo la hipótesis de participación en el mercado eléctrico diario español de energía. Se ha realizado una simulación de un año completo con el propósito de abarcar una gran variedad de condiciones meteorológicas. Se han empleado valores reales para los precios de la energía, los costes de penalización, el recurso solar y su predicción. Los resultados numéricos muestran la viabilidad de la propuesta realizada.

En la Sección 2 se plantea de forma genérica el problema. En la Sección 3 se describe la propuesta realizada. La Sección 4 presenta un caso de estudio basado en la simulación anual de una planta basada en CSP. Las conclusiones se recogen en la Sección 5 .

\section{PLANTEAMIENTO DEL PROBLEMA}

Esta sección comienza con una descripción somera de las plantas CSP. A continuación se describe el problema de generación eléctrica a resolver y finalmente se presenta la estrategia de planificación propuesta.

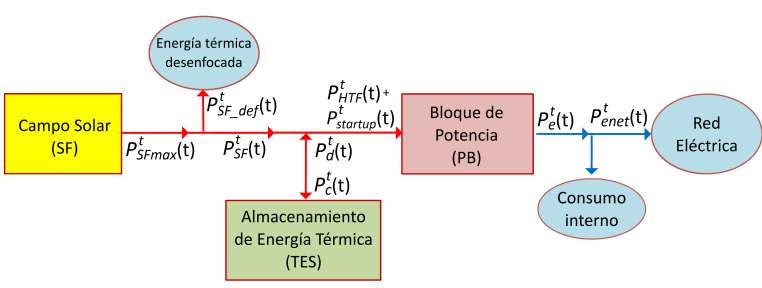

Figura 1: Esquema simplificado de una planta CSP con TES.

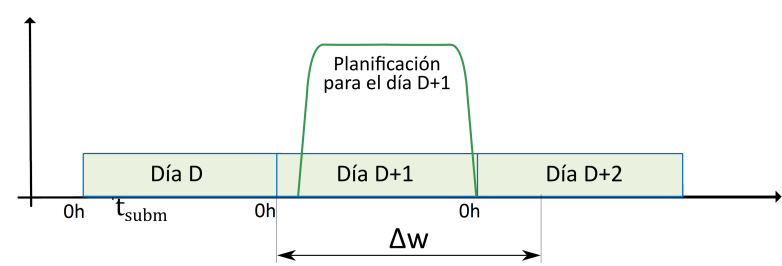

Figura 2: Ventana de planificación de la estrategia DAS.

\subsection{Breve descripción de una planta solar de concentración}

El tipo de planta solar de concentración considerada en este trabajo es una planta basada en PTC con TES. La figura 1 muestra un diagrama simplificado de esta planta, la cual consta de tres bloques principales: el SF, el PB y el TES. Aunque la presencia de un sistema de respaldo basado en combustible fósil es común en este tipo de plantas, no se considera en nuestro caso para propósitos de generación eléctrica. Los fluidos que circulan en el SF, PB y TES son HTF; HTF, agua y vapor; y HTF y sales respectivamente. Un sistema de intercambio de calor permite el intercambio de energía bidireccional entre el HTF y las sales y otro sistema intercambiador permite la transferencia energética desde SF y/o TES al PB.

La figura 1 muestra un esquema de cómo fluye la energía entre los diferentes bloques de la planta CSP. Se denota como $P_{S F \max }^{t}(t)$ a la máxima energía térmica disponible en HTF captada por el SF en el instante $t$. Con $P_{S F_{\text {def }}}^{t}(t)$ se denota la parte de esta energía que se puede perder debido a desenfoques parciales o totales de los espejos parabólicos necesarios para evitar un excesivo calentamiento del HTF debido a una demanda pequeña de energía. Por lo tanto, la diferencia entre las dos cantidades previas es la energía térmica realmente transferida desde el SF al HTF, denotada como $P_{S F}^{t}(t)$. El sistema de almacenamiento está compuesto de dos tanques de sales fundidas. La sal fundida circula desde el tanque frío (caliente) al tanque caliente (frio) en los modos de carga 
(descarga) de energía. Un intercambiador de calor bidireccional permite la transferencia entre el HTF y la sal fundida. Se denota como $P_{c}^{t}(t)$ y $P_{d}^{t}(t)$ a la potencia térmica cargada o descargada en el instante $t$ respectivamente.

El PB está constituido por un tren de intercambiadores de calor, una turbina de vapor acoplada a un generador de electricidad, un condensador, un sistema de refrigeración y otros elementos auxiliares. Se denota $P_{H T F}^{t}(t)$ a la potencia térmica a la entrada del PB usada para generar electricidad. Nótese que $P_{H T F}^{t}(t)$ se obtiene de la expresión $P_{S F}^{t}(t)+P_{d}^{t}(t)-P_{c}^{t}(t)-P_{\text {startup }}^{t}(t)$ donde $P_{\text {startup }}^{t}(t)$ es la fracción de potencia usada para incrementar el estado térmico del PB en el arranque. Finalmente $P_{\text {enet }}^{t}(t)$ es la energía eléctrica transferida a la red, descontando el consumo interno de la planta a la potencia eléctrica generada $P_{e}^{t}(t)$.

Las plantas CSP tienen varios modos de operación (por ejemplo, $\mathrm{SF} \rightarrow \mathrm{PB}, \mathrm{SF} \rightarrow \mathrm{TES}, \mathrm{SF} \rightarrow \mathrm{PB}+$ $\mathrm{TES}, \mathrm{SF}+\mathrm{TES} \rightarrow \mathrm{PB}$, TES $\rightarrow \mathrm{PB})$. Normalmente la planta es operada siguiendo una secuencia de fases. Fases operativas típicas en el SF incluyen recirculación nocturna, protección ante congelación, periodo de calentamiento del HTF o periodo operativo bajo sol entre otras.

Con el objeto de facilitar la interpretación de las variables de potencia, en lo que resta de artículo, los valores horarios medios de las diferentes potencias se denotan igual que las variables correspondientes a las potencias instantáneas pero suprimiendo el superíndice $t$.

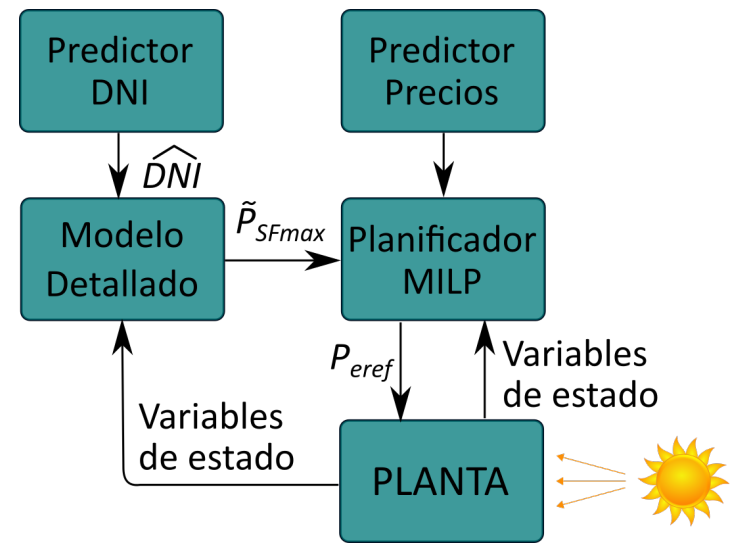

Figura 3: Diagrama de bloques estrategia DASMOD.

\subsection{Generación de potencia eléctrica en mercado eléctrico}

Esta sección describe el problema de generación eléctrica planteado. Se asume que la planta CSP participa en un mercado de compra y venta de potencia eléctrica a un día y que la producción de la planta no influye en los precios de mercado.

Un primer aspecto a considerar es la fase de planificación. Cada día $D$ (se considera que $D$ es una fecha compuesta por año, mes y día, ver figura 2) a la hora $t=t_{\text {subm }}$ el propietario de la planta CSP debe proporcionar al mercado un perfil de generación para el siguiente día $D+1$ (la notación $D+1$ indica el día siguiente a $D$ ). Este perfil que representa los valores medios horarios de la potencia generada se denota como $P_{\text {eref }}(j \mid D+1)$ con $j=1, \ldots, 24$. El objetivo de la planificación debe ser maximizar los beneficios.

Una vez el propietario se ha comprometido a proporcionar cierta potencia eléctrica, otro aspecto importante es el grado de cumplimiento alcanzado. Cada hora $j$ del día actual $(D)$, la media de la potencia generada y enviada a red, denotada $P_{\text {enet }}(j \mid D)$, debería ser igual a la potencia comprometida el día anterior, esto es $P_{\text {eref }}(j \mid D)$. Si no se da dicha igualdad, el mercado aplica penalizaciones económicas asociadas a la diferencia $P_{\text {eref }}(j \mid D)-P_{\text {enet }}(j \mid D)$.

\subsection{Estrategia de planificación a un día (DAS, day-ahead scheduling)}

Esta sección describe una estrategia clásica de planificación óptima que resuelve el problema planteado en la sección anterior y además presenta la estrategia de planificación alternativa que propone este artículo.

La planificación para el día $D+1$ se genera a la hora $t_{\text {subm }}$ del día $D$ resolviendo un modelo MILP de resolución horaria y con horizonte temporal de longitud definida por $\Delta w$ (ver figura 2). Es necesario un modelo MILP debido a que las restricciones operativas de la planta se modelan mediante variables binarias. El modelo MILP tiene como entrada un conjunto de predicciones de potencia térmica disponible, de precios futuros de venta de energía y el estado actual de la planta (necesario para estimar las condiciones iniciales del día $D+1)$. Una vez resuelto el problema de optimización, el modelo devuelve el perfil de generación del día siguiente $P_{\text {eref }}(j \mid D+1)$ con $j=1, \ldots, 24$. Las condiciones iniciales para el día $D+1$ se estiman empleando el estado actual de la planta, la predicción a un día vista y el plan de producción comprometido aún por ejecutar. Una descripción completa del modelo MILP usado está fuera del objetivo de este artículo pero puede ser consultado en [12]. Se considera un valor de $\Delta w$ superior a 24 horas con el objetivo de tener en cuenta el día $D+2$ (total o parcialmente) en el problema de optimización. La producción horaria que finalmente se realiza 
$P_{\text {enet }}(j \mid D)$, con $j=1, \ldots, 24$, es el valor máximo posible con la energía realmente disponible cada hora siempre que no supere el valor comprometido $P_{\text {eref }}(j \mid D)$ con $j=1, \ldots, 24$.

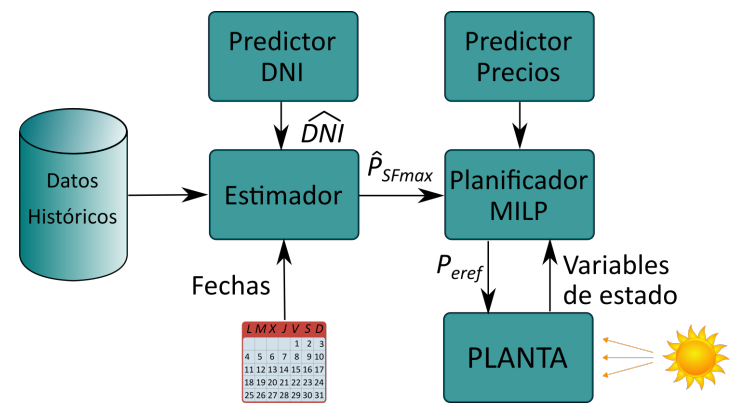

Figura 4: Diagrama de bloques estrategia DASDAT.

Con objeto de implementar la estrategia DAS, se propone el siguiente conjunto de bloques funcionales (ver figure 3 ):

- Planificador basado en MILP. Este bloque proporciona el perfil de generación del día siguiente a la hora $t=t_{\text {subm }}$ del día actual y estima las condiciones iniciales del día $D+1$.

- Modelo detallado del SF. Este bloque se utiliza para obtener las predicciones de los valores medios horarios de la máxima potencia térmica disponible en el SF $\left(\tilde{P}_{S F \max }(j / D)\right.$, con $j=t_{\text {subm }}-1, . ., 24$ y $\tilde{P}_{S F \max }(j / D+1)$, para $j=1, \ldots, \Delta w)$. El primer vector de predicciones se utiliza para estimar las condiciones iniciales de la planta en el día $D+1$ y el segundo vector de predicciones se utiliza para obtener la planificación del día $D+1$. El modelo tiene como entradas predicciones de DNI y un conjunto de variables binarias y continuas medidas en la planta CSP. Este conjunto de variables binarias y continuas se usan para caracterizar el estado actual de la planta CSP. Este trabajo ha utilizado el modelo desarrollado en [4, 12]. El modelo combina ecuaciones en diferencia y máquinas de estado para simular de forma detallada el SF.

- Predictor para el DNI y los precios de la electricidad.

Este trabajo está inspirado en la siguiente pregunta: ¿es posible proponer una estrategia tipo DAS sin necesidad de disponer de un modelo detallado del SF?. El objetivo de este trabajo es proponer un esquema alternativo (ver figura 4). En este nuevo esquema se ha sustituido el modelo detallado del SF por un sistema de estimación basado en histórico. Se asume la existencia de un histórico donde se ha almacenado el DNI diario incidente en la planta y la potencia térmica máxima obtenida del SF con esa radiación. Esos datos se deben haber recopilado mediante un conjunto de sensores instalados en el SF. Se asume que en el SF se aplica una única estrategia de operación generando un comportamiento predecible. El histórico de datos, una predicción del DNI de las horas siguientes y las fechas serán las entradas del estimador que proporcionará una predicción de la potencia térmica correspondiente. Nótese que se ha asumido que para fechas similares, el estado actual de la planta en la hora $t=t_{s} u b m$ también es similar. De todas formas, el estimador se podría mejorar incluyendo información se este tipo. Las principales ventajas de la utilización de un modelo basado en datos incluyen la sencillez de su desarrollo respecto un modelo detallado, la rápida adaptación a nuevos escenarios simplemente cambiando el conjunto de datos históricos usados y la capacidad explicativa de los resultados que proporciona al estar apoyado en situaciones acaecidas en el pasado.

\section{MODELO DEL CAMPO SOLAR BASADO EN DATOS}

Esta sección presenta el modelo basado en datos del SF propuesto en este trabajo. El modelo utiliza un algoritmo de regresión basado en los k-vecinos más cercanos ponderados como método de inferencia [5].

Se asume que se dispone de un conjunto histórico de tuplas $\left\{\left(\mathbf{D N I}_{z}, \mathbf{P}_{\text {SFmax }_{z}}, D_{z}\right)\right\}$ con $z=$ $1, \ldots, N$. Se denota $D_{z}$ a una fecha compuesta por año, mes y día. En un abuso de notación se indica $D_{z}+1$ al día siguiente a $D_{z}$. Los vectores $\mathbf{D N I}_{z}$ y $\mathbf{P}_{S F \max z}$ (asociados a la fecha $D_{z}$ ) vienen definidos por

$$
\mathbf{D N I}_{z}=\left[\begin{array}{c}
D N I\left(t_{\text {subm }}+1, D_{z}\right) \\
D N I\left(t_{\text {subm }}+2, D_{z}\right) \\
\vdots \\
D N I\left(24, D_{z}\right) \\
D N I\left(1, D_{z}+1\right) \\
D N I\left(2, D_{z}+1\right) \\
\vdots \\
\mathbf{P}_{S F \max z}=\left[\begin{array}{c}
P_{S F \max }\left(t_{\text {subm }}+1, D_{z}\right) \\
P_{S F \max }\left(t_{s u b m}+2, D_{z}\right) \\
\vdots \\
P_{S F \max }\left(24, D_{z}\right) \\
P_{S F \max }\left(1, D_{z}+1\right) \\
P_{S F \max }\left(2, D_{z}+1\right) \\
\vdots \\
P_{S F \max }\left(\Delta w, D_{z}+1\right)
\end{array}\right] .
\end{array}\right.
$$


Dado un nuevo vector de radiación predicha $\widehat{\mathbf{D N I}}$ y una fecha asociada $\hat{D}$, el objetivo es estimar la potencia térmica capturada por el SF, denotada $\widehat{\mathbf{P}}_{S F \max }$. La estimación se obtiene ponderando algunos de los vectores de potencia presentes en el histórico mediante la formula

$$
\widehat{\mathbf{P}}_{S F \max }=\sum_{l=1}^{K_{N}} p_{l} \mathbf{P}_{\text {SFmax }_{s_{l}}},
$$

donde $K_{N} \leq N$ es un hiperparámetro ajustable que define el número de vectores a ponderar, $p_{l} \geq$ 0 son un conjunto de pesos a determinar que deben cumplir $\sum_{l=1}^{K_{N}} p_{l}=1 \mathrm{y} s_{l}$ son índices tales que $s_{l} \in$ $\{1,2, \ldots, N\}$. Con el objeto de obtener $p_{l}$ se define una medida de similitud $d_{z} \in \mathcal{R}^{+}$entre $\widehat{\mathbf{D N I}}$ y $\mathbf{D N I}_{z}$ definida como:

$$
d_{z}=\exp \left(-\left\|A^{\frac{1}{2}}\left[\begin{array}{c}
\mathbf{D N I}_{z}-\widehat{\mathbf{D N I}} \\
D_{y}\left(D_{z}\right)-D_{y}(\hat{D})
\end{array}\right]\right\|_{2}\right)
$$

Nótese que $\exp (\cdot)$ es la función exponencial, y que $d_{z}$ alcanza el valor máximo de 1 cuando la coincidencia entre los dos vectores es exacta, decreciendo este valor cuando la coincidencia también decrece. Se tiene que la matriz $A^{\left(25-t_{s u b m}+\Delta w\right) \times\left(25-t_{s u b m}+\Delta w\right)}$ es diagonal, estando los elementos de la diagonal $a_{i i}$, con $i=$ $1, \ldots, 25-t_{\text {subm }}+\Delta w$, definidos mediante la expresión

$$
a_{i i} \begin{cases}\gamma_{1}, & i \leq 24-t_{\text {subm }}+\Delta w \\ \gamma_{2}, & i=25-t_{\text {subm }}+\Delta w\end{cases}
$$

donde $\gamma_{1}, \gamma_{2} \in \mathcal{R}^{+}$son dos hiperparámetros que se deben ajustar. Esos hiperparámetros balancean la importancia entre las similitudes entre radiaciones y las similitudes entre fechas. Así mismo la función $D_{y}(\cdot)$ toma como argumento una fecha y devuelve un número entero comprendido entre 1 y 183 . Este número corresponde al número del día dentro del año de la fecha si dicho número es menor o igual a 183. Si el número es mayor a 183 se devuelve la diferencia entre 365 y ese valor. Esta operación se realiza al asumir un comportamiento de la planta simétrico respecto del día 183 del año.

Finalmente se definen los pesos

$$
p_{l}=\frac{d_{s_{l}}}{\sum_{l^{\prime}=1}^{K_{N}} d_{s_{l}^{\prime}}}
$$

siendo $l=1, \ldots, K_{N}$ y $d_{s_{i}} \geq d_{s_{i+1}}$ con $i=$ $1, \ldots, N-1$.

Con el fin de diferenciar las dos estrategias de planificación a estudiar, se denotará DAS-MOD a la estrategia basada en modelo y DAS-DAT a la estrategia basada en datos. A esta última estrategia se le denotará DAS-DAT $\left(\gamma_{1}, \gamma_{2}, K_{N}\right)$ cuando se quiera hacer énfasis en los valores de los hiperparámetros utilizados.

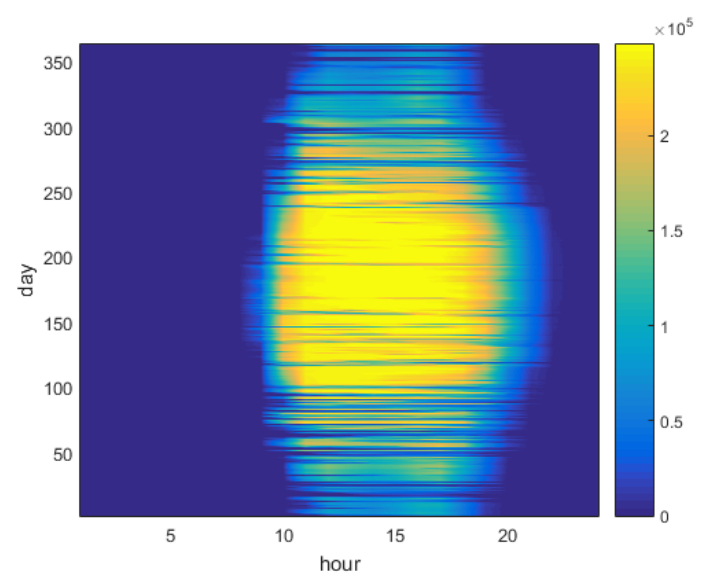

Figura 5: Valores medios horarios de la máxima potencia térmica disponible en el SF $\left(P_{S F \max }, \mathrm{kW}\right)$.

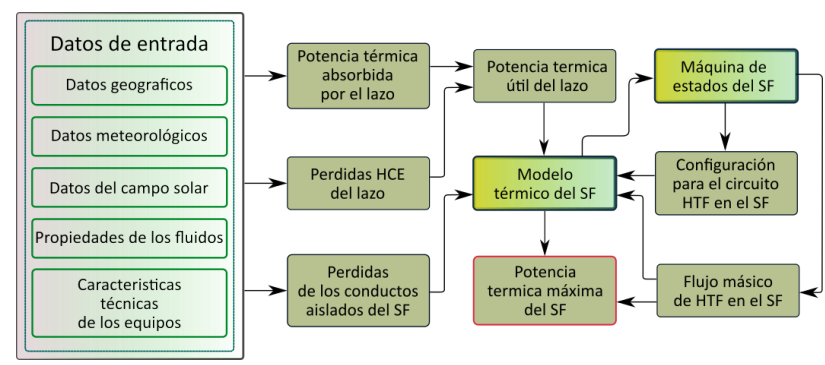

Figura 6: Diagrama de bloques del modelo detallado del SF.

\section{CASO DE ESTUDIO}

La estrategia propuesta en este trabajo se aplica, en un contexto de simulación, a una planta basada en PTC de 50 MW con almacenamiento térmico. La planta CSP analizada en este caso de estudio se basa en el modelo presentado en [4], y también usado en [12], que describe la planta Andasol 2 en Granada, España. Algunas características de este modelo (adaptadas a este caso de estudio) pueden consultarse en la Tabla 1.

Se ha considerado un periodo de una año (2013) con el propósito de testear una gran variedad de condiciones meteorológicas. Se asume la participación en el mercado diario de electricidad y la imposibilidad del productor de influir en los precios (dado su pequeño volumen de producción). El precio horario de la venta de la producción se obtiene 
Cuadro 1: Características de la planta CSP

\begin{tabular}{lr} 
Capacidad bruta de turbina & $52.5 \mathrm{MW}-\mathrm{e}$ \\
Capacidad del SF & $250 \mathrm{MW}-\mathrm{t}$ \\
Capacidad térmica en modo sólo solar & $140 \mathrm{MW}-\mathrm{t}$ \\
Capacidad térmica en modo sólo TES & $119 \mathrm{MW}-\mathrm{t}$ \\
Múltiple solar & 1.8 \\
Capacidad del TES (modo sólo TES) & 8 hours \\
Eficiencia del bloque de potencia (plena carga) & $38 \%$ \\
Sin generación eléctrica con calderas & \\
\hline
\end{tabular}

del operador de mercado ibérico OMIE, ([1]). Para diseñar un predictor de precios, se ha considerado un escenario sintético que combina el método persistente y los valores reales de los precios. El objetivo ha sido obtener un predictor con una métrica RMSE (raíz cuadrada del error cuadrático medio) de 2.7 Euros/MW h [15] para la predicción a un día. Los costes de penalización por MWh desviado respecto al plan comprometido se han obtenidos de Red Eléctrica de España. Por otra parte, los costes de generación se han supuesto despreciables por simplicidad. La resolución del plan de producción es horaria. El parámetro $t_{s u b m}$ se considera igual a 10,0 h (mercado español en 2013). El parámetro $\Delta w$ se fija a $34 \mathrm{~h}$, es decir, un esquema intermedio entre las planificaciones a un día vista y a dos días vista [14]. El DNI y la temperatura ambiente son las únicas variables meteorológicas consideradas. La predicción de temperatura ambiente se supone perfecta, se crea mediante datos TMY2 [3] y por tanto no se considera una variable de entrada en los diferentes modelos. Se dispone de un conjunto de medidas del recurso solar. Se ha generado un predictor sintético de DNI utilizando el método persistente en combinación con los valores reales. El propósito es obtener un predictor con métrica nRMSE (la raíz cuadrada el error cuadrático medio normalizado) anual del $32 \%$ [9], $43 \%$ y $45 \%$ para la predicción a uno, dos y tres días respectivamente.

En el contexto de la simulación propuesta, se sustituye la planta real CSP por una representación compuesta por dos modelos. Por un lado se utiliza de nuevo el modelo detallado del SF que caracteriza la evolución de las temperaturas y fases de operación en el SF. Dicho modelo genera los valores promedios horarios de la potencia térmica máxima disponible en el SF $\left(P_{S F \max }(j / D)\right)$, que se obtienen usando los datos de radiación solar disponible (ver figura 5). Como se observa en la figura 5, el perfil de $P_{S F \max }(j / D)$ aumenta en intensidad, longitud y estabilidad los meses centrales del año (época veraniega). Por otro lado se utiliza un modelo de resolución horaria para representar los balances energéticos y fases operativas del resto de bloques de la planta. Este esquema evita un alto tiempo de simulación, al igual que en [7, 8]. Particularmente, se emplea un modelo MILP muy similar al utilizado por el bloque planificador (ver
Cuadro 2: Métrica de error de las predicciones

\begin{tabular}{lc}
\hline Error & nRMSE \\
\hline$\hat{P}_{S F \max }-P_{S F \max }$ & $28.28 \%$ \\
$\tilde{P}_{S F \max }-P_{S F \max }$ & $29.05 \%$ \\
$\hat{P}_{S F \max }-\tilde{P}_{S F \max }$ & $12.77 \%$ \\
$D N I-\overline{D N I}$ & $40.42 \%$ \\
\hline
\end{tabular}

figura 3). Por lo tanto, es importante destacar que, en la estrategia DAS-MOD parte con cierta ventaja al utilizar un modelo de SF idéntico al usado para simular la planta real. En este caso las diferencias entre potencia térmica máxima disponible predicha y la generada en la planta sólo se deben a errores de predicción del DNI. No ocurre lo mismo en la estrategia DAS-DAT, que al utilizar un histórico para predecir la potencia térmica máxima disponible, introduce errores de modelado. Esa es la razón por la que es interesante realizar un estudio que cuantifique si esa incertidumbre dificulta la utilizad práctica del DAS-DAT.

\subsection{Modelo detallado del SF}

El SF se compone de un número de lazos PTC conectados en paralelo unos a otros. Los colectores está formados por espejos parabólicos y tubos de recepción instalados en la línea focal de la superficie parabólica. El HTF circulante fluye dentro de los tubos de recepción. Tuberías aisladas distribuyen el HTF a los diferentes lazos. Un sistema de seguimiento de un eje facilita que los colectores sigan la trayectoria del sol. Una gran cantidad de energía solar se concentra en los tubos de recepción y se transfieren al HTF. En este trabajo se ha utilizado como modelo detallado del SF el desarrollado en $[4,12]$. La figura 6 muestra el diagrama de bloques que representa el modelo. Dicho modelo está compuesto por ecuaciones dinámicas (discretizadas con un tiempo de paso de 10 segundos) y ecuaciones estáticas que representan estados de equilibrio.

\subsection{Resultados numéricos}

El conjunto de datos anuales disponible se ha dividido en dos partes. Los primeros 181 días (6 primeros meses) se han utilizado para estimar en un esquema de validación cruzada (leave-one-out) valores óptimos de los hiperparámetros. Se han obtenido los valores $\gamma_{1}=0,014, \gamma_{2}=0,24$ y $K_{N}=8$ con un error nRMSE asociado del $13.51 \%$ en la predicción de la potencia máxima disponible $P_{S F \max }$. El error es la diferencia entre la $P_{S F \max }$ real obtenida de la planta y la $\hat{P}_{S F \max }$ estimada a partir de datos, cuando se intenta predecir la potencia máxima disponible. 


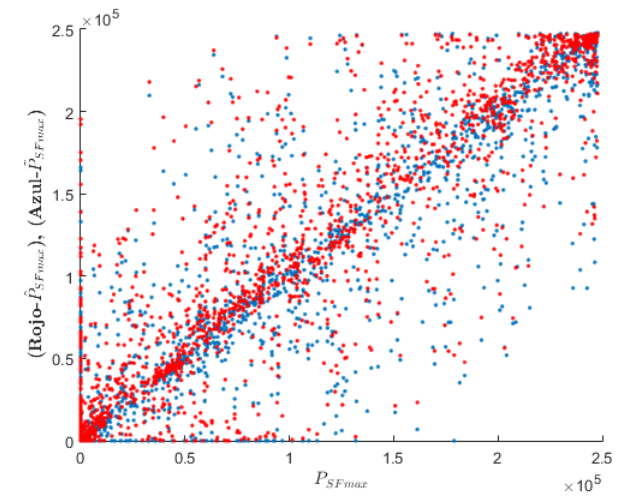

Figura 7: Comparación de $\hat{P}_{S F \max }$ y $\tilde{P}_{S F \max }$.

Cuadro 3: Resultados energéticos (6 meses)

\begin{tabular}{lccc}
\hline Valor medio horario & DAS-DAT & DAS-MOD & $\%$ \\
\hline $\begin{array}{l}\text { Potencia } \\
\text { comprometida (MWh-e) }\end{array}$ & 21.28 & 20.51 & $3.77 \%$ \\
$\begin{array}{l}\text { Potencia } \\
\text { generada (MWh-e) }\end{array}$ & 18.98 & 18.85 & $0.69 \%$ \\
$\begin{array}{l}\text { Potencia } \\
\text { desviada (MWh-e) }\end{array}$ & 2.30 & 1.66 & $38.83 \%$ \\
\hline
\end{tabular}

Los datos correspondientes a la segunda parte del año se han utilizado para probar las estrategias DAS-MOD y DAS-DAT $(0.014,0.24,8)$ y compararlas. En la figura 7 se muestran las predicciones horarias de $\tilde{P}_{S F \max }$ (basada en modelo) y $\hat{P}_{S F \max }$ (basada en datos) frente el dato real $P_{S F \max }$. Aunque ambas predicciones difieren (nRMSE del $12.77 \%$ ), el error respecto a $P_{S F \max }$ es similar (nRMSE del $29.05 \%$ y $28.28 \%$ ). La Tabla 2 resume los resultados.

En la Tabla 3 se presentan los resultados energéticos totales obtenidos en los últimos 6 meses del año para las dos estrategias estudiadas. Se observa que la estrategia DAS-DAT se compromete a generar $3.77 \%$ mas que la estrategia DAS-MOD. Este compromiso se convierte finalmente en un incremento de la producción generada de un $0.69 \%$. Esta diferencia entre lo comprometido y lo generado se traduce en un incremento de los desvíos que se cuantifica en un $38.83 \%$. Aunque las diferencias en producción no son significativas, sí lo son en el desvío, por lo que es necesario realizar un estudio económico.

En la Tabla 4 se presentan los resultados económicos para las dos estrategias estudiadas. Se aprecia que la estrategia DAS-DAT obtiene resultados muy parecidos a la estrategia DAS-MOD. Concretando, los ingresos económicos aumentan un $0,27 \%$ cuando se utiliza DAS-DAT. Por otro lado las penalizaciones económicas aumentan un 25,22\% para este último caso. Finalmente los beneficios se igualan con las dos estrategias. Se puede concluir por tanto, que ambas estrategias mantie-
Cuadro 4: Resultados económicos (6 meses)

\begin{tabular}{lcc}
\hline & DAS-DAT & DAS-MOD \\
\hline Ingresos & $€ 4.531 .394$ & $€ 4.518 .890$ \\
Penalizaciones & $€ 75.130$ & $€ 59.998$ \\
Beneficios & $€ 4.459 .139$ & $€ 4.458 .891$ \\
\hline
\end{tabular}

nen un comportamiento económico similar. Nótese que las penalizaciones por desvío que aplica el mercado eléctrico varían cada hora. Eso explica que cierto incremento en los desvíos no implica el mismo incremento en penalizaciones.

Una cuestión interesante es averiguar cómo influyen las penalizaciones por desvíos en los rendimientos económicos obtenidos por las dos alternativas propuestas. La figura 8 aborda este asunto. Se ha considerado un factor $\mu \geq 0$ que multiplica las penalizaciones por desvío. La línea negra sólida representa el incremento porcentual en beneficios que obtiene la estrategia DAS-DAT respecto la estrategia DAS-MOD. Se observa que en el rango $\mu \in[0,3]$ la variación entre ambas estrategias es menor al $1 \%$ manteniéndose la equivalencia entre las dos estrategias a pesar de considerables variaciones en las penalizaciones por desvío. Como elemento comparativo se ha incluido la línea sólida roja, que representa el incremento porcentual en beneficios de la estrategia DAS-MOD respecto a sí misma para el caso $\mu=1$. En el mismo rango de variación de $\mu$ el decremento en el beneficio supera el $2,5 \%$.

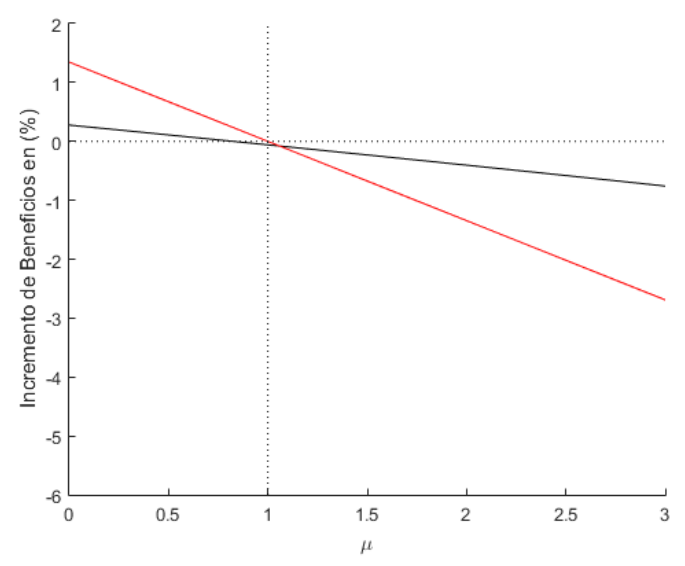

Figura 8: Beneficios vs $\mu$.

Como conclusión se tiene que la simulación muestra la posibilidad de sustituir el modelo detallado del SF por un modelo basado en datos con el objeto de implementar una estrategia DAS para participar en un mercado eléctrico. Notar que el modelo basado en datos se nutre de medidas realizadas en planta, eludiendo la complejidad de la creación de un modelo detallado. 


\section{Conclusiones}

La participación de plantas CSP en mercados eléctricos requieren el diseño de una estrategia de planificación. Un elemento importante de esa estrategia es un modelo del campo solar que proporcione información sobre la potencia térmica disponible. Este modelo se puede obtener mediante un diseño detallado utilizando los principios físicos que rigen la planta. Sin embargo, este trabajo plantea una alternativa que consiste en diseñar un modelo basado directamente en datos históricos de la planta. En este caso la potencia térmica disponible en el campo solar se obtiene a partir del recurso solar presente, medidas históricas de potencia generadas en planta y un algoritmo de predicción. El trabajo presenta un caso de estudio simulado que considera la participación en el mercado diario español de una planta tipo CSP de $50 \mathrm{MW}$. Se han considerado datos anuales reales de recurso solar, precio de venta de la electricidad y costes de los desvío para realizar una comparativa entre las dos estrategias. La conclusión es que la estrategia basada en datos es competitiva respecto la estrategia basada en modelo detallado, lo que facilita el diseño de una estrategia de planificación para la participación en mercados eléctricos.

\section{Agradecimientos}

Este trabajo ha sido financiado por el proyecto DPI2016-76493-C3-2-R del MINECO (España).

\section{Referencias}

[1] OMIE. Last access: 28.04.17. http://www . omie.es/, 2017.

[2] The SAM website. Last access: 28.04.17. https://sam.nrel.gov/, 2017.

[3] User's manual for TMY2s. Last access: 28.04.17. http://rredc.nrel.gov/solar/ pubs/tmy2/, 2017.

[4] Isabel Llorente Garcia, Jose Luis Alvarez, and Daniel Blanco. Performance model for parabolic trough solar thermal power plants with thermal storage: Comparison to operating plant data. Solar Energy, 85(10):2443 2460, 2011.

[5] Trevor Hastie, Robert Tibshirani, and Jerome Friedman. The elements of statistical learning: data mining, inference and prediction. Springer, 2 edition, 2009.

[6] Christoph Kost, Christoph M. Flath, and Dominik Most. Concentrating solar power plant investment and operation decisions under different price and support mechanisms. Energy Policy, 61(0):238 - 248, 2013.

[7] Birk Kraas, Marion Schroedter-Homscheidt, and Reinhard Madlener. Economic merits of a state-of-the-art concentrating solar power forecasting system for participation in the Spanish electricity market. Solar Energy, 93(0):244 - 255, 2013.

[8] Edward W. Law, Merlinde Kay, and Robert A. Taylor. Calculating the financial value of a concentrated solar thermal plant operated using direct normal irradiance forecasts. Solar Energy, 125:267 - 281, 2016.

[9] Edward W. Law, Abhnil A. Prasad, Merlinde Kay, and Robert A. Taylor. Direct normal irradiance forecasting and its application to concentrated solar thermal output forecasting — A review. Solar Energy, 108(0):287 307, 2014.

[10] R. Sioshansi and P. Denholm. The value of concentrating solar power and thermal energy storage. Sustainable Energy, IEEE Transactions on, 1(3):173-183, Oct 2010.

[11] J. Usaola. Operation of concentrating solar power plants with storage in spot electricity markets. Renewable Power Generation, IET, 6(1):59-66, January 2012.

[12] Manuel Jesús Vasallo and José Manuel Bravo. A novel two-model based approach for optimal scheduling in CSP plants. Solar Energy, 126:73 - 92, 2016.

[13] Manuel Jesús Vasallo and José Manuel Bravo. A MPC approach for optimal generation scheduling in CSP plants. Applied Energy, 165:357 - 370, 2016.

[14] Michael Wittmann, Markus Eck, Robert Pitz-Paal, and Hans Muller-Steinhagen. Methodology for optimized operation strategies of solar thermal power plants with integrated heat storage. Solar Energy, 85(4):653 - 659, 2011. SolarPACES 2009.

[15] Zhang Yang, Li Ce, and Li Lian. Electricity price forecasting by a hybrid model, combining wavelet transform, arma and kernelbased extreme learning machine methods. Applied Energy, 190(Supplement C):291 305, 2017.

[16] H.L. Zhang, J. Baeyens, J. Degréve, and G. Cacéres. Concentrated solar power plants: Review and design methodology. Renewable and Sustainable Energy Reviews, 22(0):466 481, 2013. 